\title{
LA HABITABILIDAD EFÍMERA: EL ESPACIO PÚBLICO COMO REFUGIO DE DESASTRES EN CIUDAD DE MÉXICO
}

Habitabilidade efêmera: espaço público como refúgio de desastres na Cidade do México

Ephemeral habitability: Public space as a refuge from disasters in Mexico City

\section{Milton Montejano Castillo}

Profesor e investigador en la Sección de Estudios de Posgrado e Investigación Escuela Superior de Ingeniería y Arquitectura, Unidad Tecamachalco Miembro del Sistema Nacional de Investigadores de CONACYT (SNI-I). Instituto Politécnico Nacional, Ciudad de México, México.

mmontejanoc@ipn.mx

https://orcid.org/0000-0002-5804-2364

\section{Mildred Moreno Villanueva}

Investigadora de la Escuela Superior de Ingeniería y Arquitectura, Unidad Tecamachalco - Miembro del Sistema Nacional de Investigadores de CONACYT (SNI-I). Instituto Politécnico Nacional, Ciudad de México, México. mmorenov@ipn.mx http://orcid.org/0000-0001-5835-8572

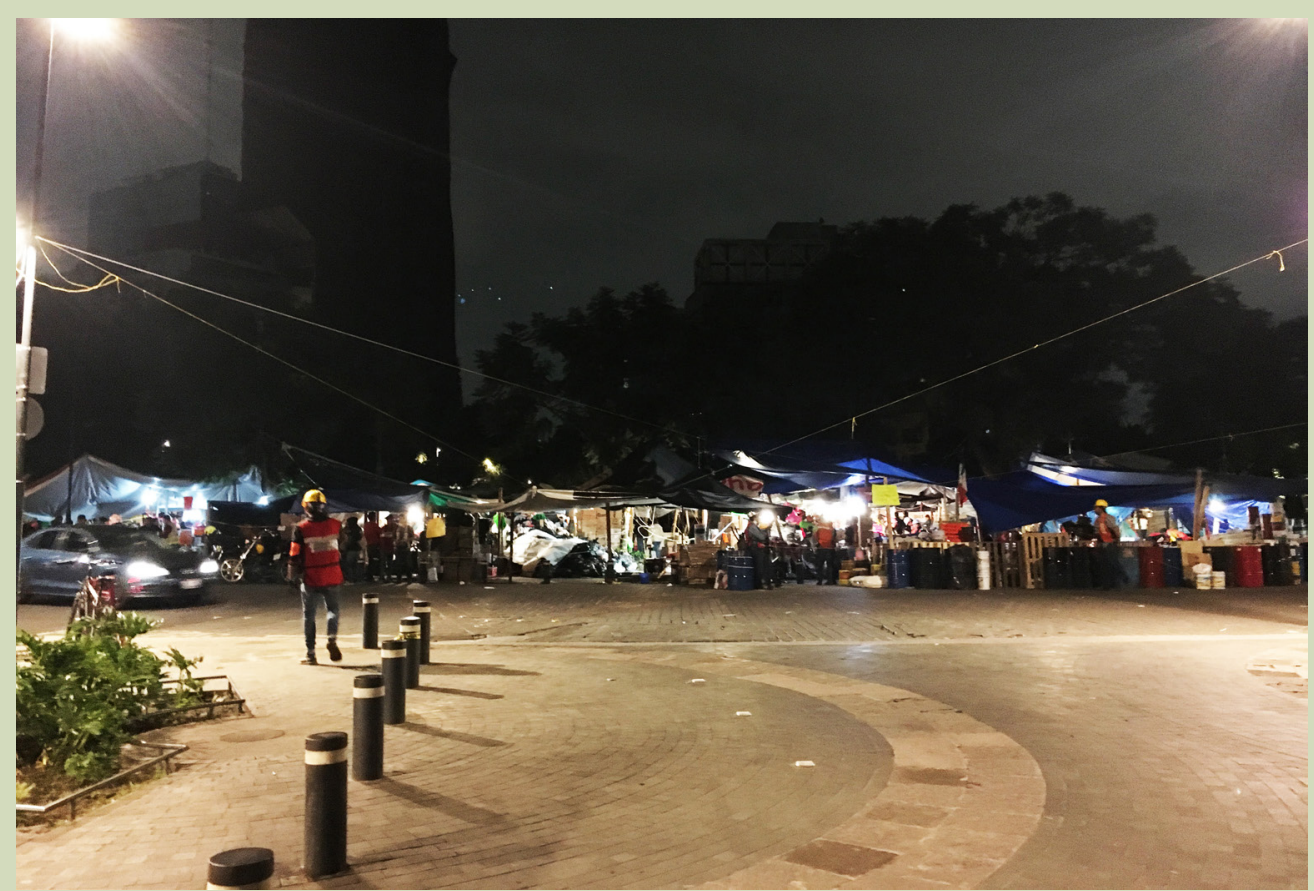

Este es un subproducto de los proyectos de investigación IPN-SIP 20181220 y 20161821 financiados por la Secretaría de Investigación y Posgrado del Instituto Politécnico Nacional, México.

Miembros del

Ejército mexicano construyen un espacio provisional de apoyo en una de las calles aledañas a la zona con mayores daños durante el sismo de septiembre de 2017 en Ciudad de México. Fuente: Autores. 


\title{
RESUMEN
}

En el imaginario colectivo, el concepto de habitabilidad se ha concebido principalmente como una característica fija y permanente de un espacio construido. Sin embargo, en las ciudades con riesgo de desastre, ciertas condiciones emergentes han requerido que espacios -en principio no construidos- se deban convertir de manera súbita en espacios habitables en su sentido más amplio, como sería el uso de espacios públicos como refugio en las etapas de emergencia y recuperación ante desastres. Lo anterior ha dado lugar, de manera espontánea o planificada, a la construcción de una habitabilidad efímera en el espacio público. Sin embargo, en el camino para lograr esa condición existen muchos factores de diversa naturaleza que van desde las acciones para evitar la desaparición de espacios públicos debido a la privatización hasta una buena coordinación de programas para lograr esa habitabilidad. En este artículo se analiza la mayor cantidad posible de esos factores a partir de un análisis de fortalezas, oportunidades y amenazas para el caso de Ciudad de México. Los resultados indican que, ante una amenaza constante de privatización del espacio público, la habitabilidad de esos espacios se ha estado promoviendo a través de programas y diversos instrumentos, no obstante de manera dispersa y sin una continuidad en el tiempo. Por otra parte, a pesar de las debilidades y amenazas detectadas, se identifican fortalezas y oportunidades que pueden servir como base para la formulación de estrategias para hacer más habitables los espacios públicos durante una situación posdesastre, para una de las ciudades con mayor población expuesta a desastres de ese país.

Palabras clave: espacio público, catástrofes, gestión urbana, arquitectura efímera, construcción para emergencias.

\section{RESUMO}

Na imaginação coletiva, o conceito de habitabilidade foi concebido principalmente como uma característica fixa e permanente de um espaço construído. No entanto, nas cidades em risco de desastre, certas condições emergentes exigiram que os espaços - inicialmente não construídos - se tornassem subitamente habitáveis em seu sentido mais amplo, como o uso de espaços públicos como abrigos em estágios de emergência e recuperação de desastres. O precedente deu origem, espontânea ou planejada, à construção de uma habitabilidade efêmera no espaço público. No entanto, na maneira de alcançar essa condição, existem muitos fatores de natureza diversa, que vão desde ações para evitar o desaparecimento de espaços públicos devido à privatização até uma boa coordenação de programas para alcançar essa habitabilidade. Este artigo analisa o maior número possível desses fatores com base em uma análise de pontos fortes, oportunidades e ameaças no caso da Cidade do México. Os resultados indicam que, diante de uma constante ameaça de privatização do espaço público, a habitabilidade desses espaços foi promovida por meio de programas e diversos instrumentos, embora de maneira dispersa e sem continuidade no tempo. Por outro lado, e apesar das fraquezas e ameaças detectadas, são identificados pontos fortes e oportunidades que podem servir de base para a formulação de estratégias para tornar os espaços públicos mais habitáveis durante uma situação pós-desastre, para uma das cidades com a maior população exposta a desastres daquele país.

Palavras chave: espaço público, catástrofes, gestão urbana, arquitetura efêmera, construção de emergência.

\begin{abstract}
In the collective imaginary, the concept of habitability has been conceived mainly as a fixed and permanent feature of a built space. However, in cities with disaster risks, certain emerging conditions have required that spaces -in principle undeveloped-should suddenly become habitable spaces in their broadest sense, such as the use of public spaces as a shelter during emergencies and disaster recovery. This condition has given rise, spontaneously or in a planned way, to the construction of an ephemeral habitability in the public space. However, in the road to achieve that condition, there are many diverse factors ranging from actions to prevent the disappearance of public spaces due to privatization, to a good coordination of programs to achieve that habitability. This article analyzes as many of those factors as possible, based on an analysis of strengths, opportunities and threats for the case of Mexico City. The results indicate that in the face of a constant threat of privatization of public space, the habitability of these spaces has been promoted through programs and different instruments, but in a scattered way and without continuity over time. On the other hand, despite the weaknesses and threats detected, strengths and opportunities are identified that can serve as a basis for formulating strategies to make public spaces more habitable during a post-disaster situation, for one of that country's highest populated disaster exposed cities.
\end{abstract}

Keywords: public space, disasters, urban management, ephemeral architecture, construction for emergencies. 


\section{INTRODUCCIÓN: EL ESPACIO PÚBLICO ANTE DESASTRES COMO OBJETO DE INVESTIGACIÓN}

1 Aunque en la literatura relacionada con este tema se utiliza el término "espacio abierto", para efectos de este artículo consideramos el espacio abierto como parte de la definición de espacio público, pues de acuerdo a Lofland (en Delgado, 2011: 17-18), por espacio público podemos entender "Aquellas áreas de una ciudad a las que, en general, todas las personas tienen acceso legal....las calles de la ciudad, sus parques, sus lugares de acomodo públicos...los edificios públicos o a las 'zonas públicas' de edificios privados".

2 Entendiendo el término "resiliencia" como la capacidad que tiene un determinado territorio y los elementos que lo componen, de adaptarse a circunstancias adversas posteriores a un desastre.

3 Se refiere a la cuantificación de superficies perdidas a lo largo del tiempo susceptibles de ocuparse en caso de emergencia y que representan un recurso muy importante en ciudades en riesgo de desastre (Turer, 2015, pp. 729-742; Mafi et al., 2012, pp. 302-313).
En años recientes se ha venido cuestionando si la forma urbana puede facilitar el proceso de recuperación de una ciudad después de un desastre y en particular se ha demostrado' que el espacio público puede llegar a jugar un papel crucial en la etapa de recuperación (Allan, et al., 2013, pp. 242-262).

Considerando el espacio público como un recurso fundamental en las ciudades en riesgo de desastre, se pueden identificar diferentes temas al respecto como son: la integración entre el diseño urbano y la teoría de la resiliencia ${ }^{2}$ al considerar los espacios abiertos como una "segunda ciudad" en la recuperación ante desastres (Allan y Bryant, 2014); el análisis longitudinal ${ }^{3}$ de espacios públicos disponibles en ciudades propensas a terremotos (Turer, 2015, pp. 729-742; Mafi et al., 2012, pp. 302-313); el análisis geográfico de espacios abiertos utilizados en desastres y su relación con la forma urbana (Villagra, et al., 20 14, pp. 64-78); la construcción de escenarios de desastre es otro de los temas, donde se considera el espacio público como área de refugio en caso de posibles terremotos, utilizando criterios cuantitativos como la estimación de la demanda de espacio, la idoneidad de los espacios y accesibilidad (Anhorn y Khazai, 20 I5, pp. 789-803; Zhao et al., 2017, pp. 1-20); los nuevos espacios públicos construidos después de los terremotos como una forma de innovación y participación comunitaria (Bryant y Allan, 2013), o la significación y la re-significación del espacio público ante desastres (Berroeta, et al., 2016, pp. 143- 170; Webb, 2007, pp. 430-440).

Aunque todos estos estudios remiten a pensar el espacio público como habitable, en la política urbana y en los estudios académicos generalmente el espacio público no se concibe como un espacio habitable, sino más bien como un espacio de transición, y los estudios sobre habitabilidad del espacio público en esas circunstancias son muy escasos. Aunque diversos autores consideran que la habitabilidad se refiere únicamente a las condiciones materiales y estructurales de los espacios construidos sin tomar en cuenta el aspecto social al exterior, por lo que para efectos de este texto, se considera que la habitabilidad para el hombre sería tanto dentro del elemento arquitectónico como fuera de él. Dicha habitabilidad en el espacio público también se construye por necesidad en los momentos menos inesperados.

México, al ubicarse en una zona de interacción entre dos placas tectónicas (la Placa de Cocos y la Placa de Norteamérica), se encuentra en una zona de actividad sísmica muy elevada (Meli, 2002, pp. 125- I46). Esto hace que después del riesgo de inundaciones, el territorio sujeto a riesgo sísmico en México corresponda a más de 540,000 Km2, lo que representa casi la tercera parte del territorio nacional y casi la tercera parte (3I millones en 2010) de la población expuesta a este tipo de riesgo (SEGOB, 2012, p. 14). De esos 31 millones, más de 20 millones de habitantes están concentrados en la Zona Metropolitana de la Ciudad de México (ZMCM). 


\section{METODOLOGÍA: \\ LA HERRAMIENTA FODA PARA EL ANÁLISIS DEL ESPACIO PÚBLICO EN UN CONTEXTO DE RIESGO SÍSMICO}

Entre los sismos más recientes se encuentra el del 19 de septiembre de 2017, un terremoto de magnitud 7. I a las 13:1 4 horas con epicentro en los estados de Morelos y Puebla, que causó un total de 369 víctimas fatales en todo el país y graves daños en varias ciudades de la República Mexicana. Este evento recordó a la sociedad mexicana que la habitabilidad, si bien de manera improvisada, es una necesidad y debe continuar después de ocurrido un desastre, aún en el espacio público. Por lo tanto, este artículo tiene como objetivo explorar los factores que facilitan o limitan las condiciones de habitabilidad en el espacio público en situaciones de emergencia en la Ciudad de México. Partimos de la hipótesis de que la disponibilidad y habitabilidad del espacio público se ha basado en la adopción de tendencias apoyadas por la economía, más que en las necesidades de la población, lo que resulta en espacios públicos exclusivos e inhabitables en caso de desastre.

Por su complejidad, cada uno de los temas que asocian espacio público y desastres mencionados anteriormente, se ha estado trabajando prácticamente de manera separada, por lo que en este artículo se intentan integran bajo una sola metodología de análisis. Considerando los múltiples factores que inciden tanto en la conservación como en la gestión y adaptación del espacio público en una ciudad en riesgo de desastre, en esta sección se presenta la metodología FODA con el fin de integrar tanto las fortalezas, las oportunidades, debilidades y amenazas alrededor del espacio público como recurso ante desastres en la Ciudad de México a partir de los sismos ocurridos en esa ciudad con el objetivo de formular estrategias para el futuro de la gestión del espacio público.

El análisis FODA tradicionalmente se ha considerado como una herramienta de estudios prospectivos que ayuda a identificar problemas y oportunidades para crear escenarios. FODA es la abreviación de: a) Fortalezas, que son internas y positivas; b) Debilidades, que son internas y negativas, c) Oportunidades, que son externas y positivas, y finalmente d) Amenazas, que son externas y negativas. Una vez que se identifican los elementos descritos, todos y cada uno de los elementos se cruzan entre sí para formular estrategias a partir de las siguientes preguntas: Fortalezas contra Oportunidades: ¿Cuál de las fortalezas puede ser usada para maximizar las oportunidades identificadas? Debilidades contra Oportunidades: ¿Qué acciones se pueden tomar para minimizar las debilidades identificadas? Fortalezas contra Amenazas: ¿Cómo se puede hacer uso de las fortalezas para minimizar las amenazas? Y Debilidades contra Amenazas: ¿Cómo se pueden minimizar las debilidades para evitar las amenazas identificadas?

Para la formulación de cada uno de las categorías del análisis FODA se recurrió a referencias tanto bibliográficas como hemerográficas y visuales a partir del análisis de fotografías y videos para 
4 Proyectos de investigación IPN-SIP 20181220 y 20161821, financiados por la Secretaría de Investigación y Posgrado del Instituto Politécnico Nacional, México, que tienen el objetivo de analizar las transformaciones urbanas en zonas sujetas a riesgo sísmico en Ciudad de México, con el fin de entender mejor el papel que desempeñan los espacios abiertos después de un desastre, así como documentar las innovaciones introducidas en la forma urbana en ciudades en proceso de reconstrucción con el fin de aportar en lo posible, directrices para el proceso posdesastre en México. Esta investigación se ha estado llevando a cabo en la franja que bordea el Océano Pacífico, llamada "Cinturón de Fuego" y donde ocurre la mayor actividad sísmica del planeta. documentar los usos del espacio público durante desastre de 1985. Para documentar el uso del espacio público en 2017 se realizó un estudio de campo para corroborar cuántos y cuáles de los espacios utilizados en 1985 se volvieron a ocupar en 2017. Para los aspectos de la gestión del espacio público se hizo un análisis de los programas que se han elaborado para ese fin en las últimas décadas. Aunque parezca una metodología muy sencilla, ésta puede ser una forma muy conveniente de clasificar aspectos documentales e históricos en un esquema analítico para poder derivar estrategias y se trata de una herramienta que, a pesar de su efectividad, se ha utilizado escasamente en los estudios de arquitectura y urbanismo. Las fortalezas, debilidades, oportunidades y amenazas se basan en los resultados de proyectos de investigación realizados previamente por los autores.

Al hablar de la habitabilidad asociada a espacio público y desastres entran en juego muchos factores que van desde la pugna por evitar la desaparición del espacio público por causa de la privatización, hasta la necesaria coordinación de programas y participación de la población para adaptar y preparar espacios para una situación emergente. Considerando los múltiples factores que inciden en este fenómeno, y con el objetivo de simplificar una realidad que en sí ya es muy compleja, se optó por utilizar la herramienta de matriz FODA, que tiene como finalidad hacer una aproximación de las variables significativas que influyen sobre un objeto de estudio (Gándara et al, 2012, pp. 323-338). Las fortalezas, las oportunidades, debilidades y amenazas alrededor del espacio público como recurso ante desastres en Ciudad de México, han sido identificadas a partir de un proyecto de investigación de larga duración ${ }^{4}$ realizado por los autores, para lo cual se recurrió a referencias bibliográficas y visuales como fotografías y videos para documentar los usos del espacio público, como en el caso del sismo de 1985. 
RESULTADOS:

FORTALEZAS, OPORTUNIDADES, DEBILIDADES Y AMENAZAS DEL ESPACIO PÚBLICO ANTE DESASTRES EN CIUDAD DE MÉXICO
Con la intención de ofrecerle al lector una lectura más fluida, los títulos individuales del análisis FODA se indican únicamente al pie de página, al final de cada párrafo y el esquema completo se muestra a continuación [Tabla I].

Tabla 1 .

Análisis FODA para la elaboración de estrategias para conservar y promover la habitabilidad del espacio público ante desastres en la Ciudad de México. Elaboración: autores

\begin{tabular}{|c|c|c|}
\hline & $\begin{array}{l}\text { Oportunidades (externas / positivas) } \\
\text { (O1) Creación de nuevos espacios públicos } \\
\text { después de desastres. } \\
\text { (O2) La desindustrialización como una } \\
\text { oportunidad para crear nuevos espacios } \\
\text { públicos. } \\
\text { (O3) Las universidades como recurso huma- } \\
\text { no para la adaptación de espacios públicos } \\
\text { ante desastres. }\end{array}$ & $\begin{array}{l}\text { Amenazas (externas / negativas) } \\
\text { (A1) Incremento de la privatización del } \\
\text { espacio público utilizado durante los } \\
\text { desastres. } \\
\text { (A2) Nuevos conflictos sobre espacios } \\
\text { públicos que dejó el desastre. } \\
\text { (A3) Verticalización indiscriminada. }\end{array}$ \\
\hline $\begin{array}{l}\text { Fortalezas: (internas / positivas) } \\
\text { (F1) Una función comprobada de } \\
\text { los espacios públicos en la ciudad } \\
\text { histórica y moderna como recurso } \\
\text { durante las etapas de emergencia. } \\
\text { (F2) Protección del patrimonio de los } \\
\text { espacios públicos pertenecientes a la } \\
\text { ciudad histórica. } \\
\text { (F3) Existencia de actores y pro- } \\
\text { gramas para la gestión del espacio } \\
\text { público. }\end{array}$ & $\begin{array}{l}\text { Estrategias de fortalezas-oportunidades } \\
\text { (F1-F3 / O3) Involucrar a las universida- } \\
\text { des, el gobierno y la sociedad civil para } \\
\text { la adaptación de los espacios públicos en } \\
\text { relación con los monumentos históricos y } \\
\text { modernos. } \\
\text { (F3 / O3) Preparar espacios públicos para } \\
\text { desastres en áreas más específicas. } \\
\text { (F3 / O2) Definir proyectos en zonas desin- } \\
\text { dustrializadas de acuerdo con la naturaleza } \\
\text { sísmica del área. }\end{array}$ & $\begin{array}{l}\text { (F3-A1) Regulación del uso de espacios } \\
\text { públicos ya privatizados como parte de } \\
\text { los programas de recuperación. } \\
\text { (F3-A2) Establecimiento de un diálogo } \\
\text { más organizado entre la sociedad civil y } \\
\text { las autoridades. } \\
\text { (F2-A3) Reforzamiento de directrices para } \\
\text { la protección del patrimonio. }\end{array}$ \\
\hline $\begin{array}{l}\text { (D1) Los espacios públicos del siglo } \\
\text { XX carecen de protección coercitiva. } \\
\text { (D2) Programas de espacio público } \\
\text { no coordinados o no están bien } \\
\text { implementados. } \\
\text { (D3) No existe una continuidad de } \\
\text { autoridades para la gestión del espa- } \\
\text { cio público. }\end{array}$ & $\begin{array}{l}\text { (D2 / O2-O3) Desarrollar programas mejor } \\
\text { coordinados para la adaptación de espa- } \\
\text { cios públicos. } \\
\text { (D2 / O3) Involucrar universidades para } \\
\text { la producción de ideas de adaptación de } \\
\text { espacios públicos ante desastres. } \\
\text { (D2 / O2) Coordinar acciones entre muni- } \\
\text { cipios. }\end{array}$ & $\begin{array}{l}\text { (D2 / A1) Fortalecer y coordinar mejor la } \\
\text { protección del espacio público para que } \\
\text { no se privaticen. } \\
\text { (D3-A3) Un organismo independiente de } \\
\text { los períodos políticos. } \\
\text { (D3 / A1-A3) Fortalecer la opinión pública } \\
\text { para defender el espacio público y evitar } \\
\text { la verticalización indiscriminada. }\end{array}$ \\
\hline
\end{tabular}




\section{FORTALEZAS 5}

5 Se entiende fortaleza como los elementos internos y positivos que representan recursos y circunstancias que favorecen el uso y apropiación del espacio público en caso de emergencia.

6 Fortaleza-1 (F1). Una función comprobada de los espacios públicos en la ciudad histórica y moderna como recurso durante las etapas de emergencia.

7 Fortaleza-2 (F2). Protección del patrimonio de los espacios públicos pertenecientes a la ciudad histórica.
En 1985 y en 2017, el espacio público desempeñó un papel fundamental tanto en la fase de emergencia como en la etapa de reconstrucción [Figura I].A manera de ejemplo, para 1985 se identificaron treinta y cuatro espacios públicos utilizados inmediatamente después de ocurrido el sismo, en donde se realizaron más de diez actividades diferentes dentro de plazas, jardines, glorietas, banquetas, y camellones entre otros. Dentro de las actividades se encuentran campamentos improvisados; organización de actividades de búsqueda y rescate; espacios. de expresión de demandas sociales relacionadas con el desastre; preparación de comida; puestos de socorro y ayuda médica y psicológica; centros de acopio y reparto de donaciones; centros de información para buscar sobrevivientes; identificación de víctimas fatales y lugares de conmemoración después del desastre (Montejano y Moreno, 2016, pp. 104-1 I3). Cabe mencionar que en un espacio se llevó a cabo más de una actividad. La mayoría de esos lugares se organiza de manera espontánea tanto en la dimensión pública como institucional. Las plazas han sido los ejemplos con mayor capacidad para adaptarse a los diferentes usos, identificándose hasta ocho usos diferentes en ellas [Figura 2], aunque también otros espacios debido a sus particularidades permitieron otras funciones muy específicas, como las estaciones y túneles de metro para desplazarse por debajo de zonas colapsadas. ${ }^{6}$

Entre 1985 y 2017 , uno de los factores que permitió la conservación de muchos de esos espacios fue su naturaleza patrimonial, cuando el espacio público muestra características y valores específicos históricos y para los cuales existen instancias para su conservación o regeneración como el INAH (Instituto Nacional de Antropología e Historia), el INBA (Instituto Nacional de Bellas Artes), la Autoridad del Centro Histórico o la UNESCO, según el caso.

Como consecuencia, los espacios públicos considerados patrimonio, mantienen sus características físicas y constructivas, lo que les permite ocuparse en emergencias para diversos usos. Estas fortalezas que constituyen los espacios públicos patrimoniales se refieren a las dimensiones del lugar, la infraestructura, la ubicación, y mobiliario urbano, que en conjunto son más amplios que los actuales espacios públicos [Figura 3]. Con respecto a la organización del Centro Histórico de Ciudad de México, existe otra entidad descentralizada llamada Autoridad del Centro Histórico, creada en 2007, que propone políticas públicas de integración y promueve la renovación de los espacios públicos ubicados en este gran espacio histórico?

Además de las características patrimoniales que los espacios tienen dentro de sí, ha habido diferentes actores y programas que han pugnado por su conservación y mantenimiento, como la Secretaría de Desarrollo Urbano y Vivienda (SEDUVI), la extinta Autoridad del Espacio Público (AEP), o las diferentes alcaldías. La AEP se creó en 2008 y funcionó hasta 2018 en varios proyectos involucrando también al sector privado. Entre los proyectos de espacios públicos recientemente creados se encontraban proyectos como bajo puentes o parques públicos de bolsillo, que se refiere a espacios de nueva creación en lugares residuales de la ciudad, 

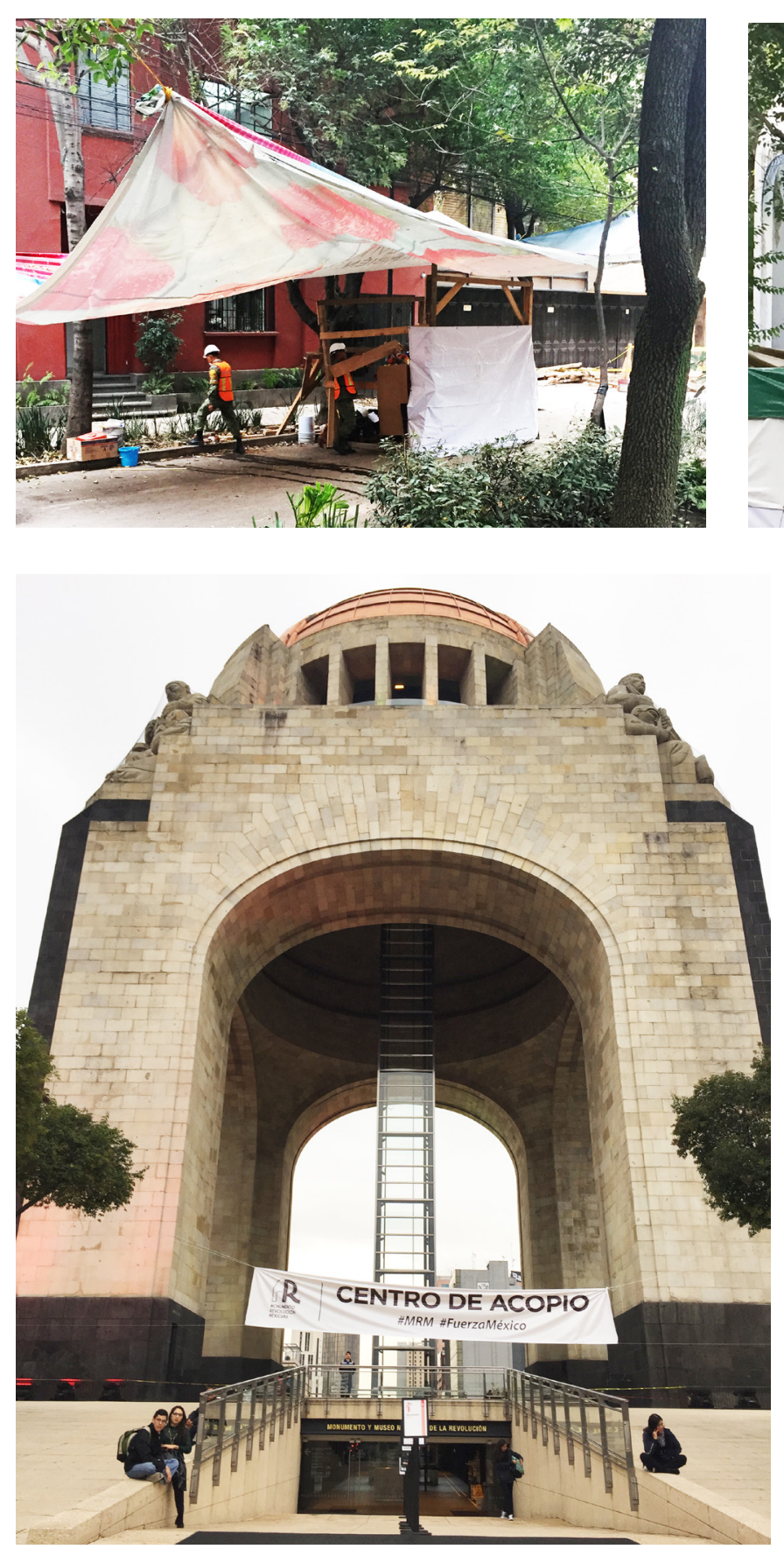

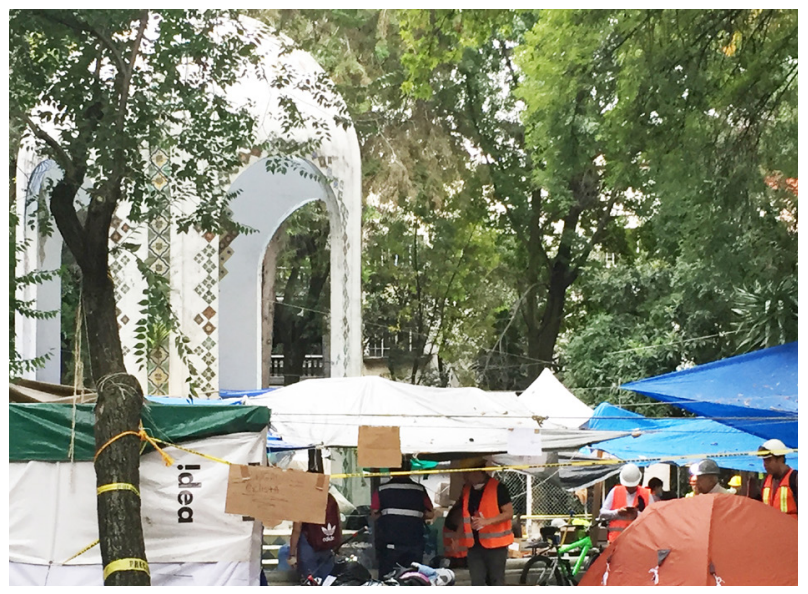

Figura 1

Miembros del Ejército mexicano construyen un espacio provisional de apoyo en una de las calles aledañas a la zona con mayores daños durante el sismo de septiembre de 2017 en Ciudad de México. Fuente: Autores.
Figura 2

Espacios improvisados para dormir y para organizar labores de búsqueda y rescate en los días siguientes al sismo de septiembre de 2017, Plaza Popocatépetl, Colonia Hipódromo Condesa, Ciudad de México. Fuente: Autores.

Figura 3

Centro de acopio

instalado en el

Monumento a la Revo-

lución, que origi-

nalmente se planeó

para ser el Palacio

Legislativo en 1910 , pero cuya realiza-

ción fue interrumpida por la Revolución Mexicana, decretándosele su uso como mausoleo desde 1938. Fotografía: Autores.

que oscilan entre 100 y 400 metros cuadrados. Por otro lado, estaban los proyectos de mejora y rehabilitación de espacios públicos, en los que se encontraban las mejoras de espacios con un diseño inclusivo, la rehabilitación de espacios patrimoniales, peatonalización y semi-peatonalización de calles, programas de iluminación y remodelaciones de monumentos. Indudablemente otro de estos actores es la sociedad civil, como uno de los recursos humanos más importantes para llevar a cabo estas acciones en coordinación con las autoridades. ${ }^{8}$
8 Fortaleza-3 (F3). Existencia de actores y programas para la gestión del espacio público. 


\section{DEBILIDADES 9}

9 Se entiende debilidad como la ausencia o carencia de recursos y circunstancias internas y negativas que impiden el aprovechamiento, el uso y la apropiación del espacio público en caso de emergencia.

10 Debilidad-1 (D1). Los espacios públicos del siglo XX carecen de protección coercitiva.

11 Los Bajo Puentes fue otro de los programas del Gobierno del Distrito Federal para la recuperación de espacios públicos abandonados para convertirlos en sitios seguros y bien iluminados para la convivencia ciudadana; su principal objetivo era ofrecer a los ciudadanos alternativas de movilidad y opciones de sano esparcimiento, convivencia social y familiar según la definición de la Secretaría de Desarrollo Urbano y Vivienda (SEDUVI).

12 Debilidad-2 (D2). Programas espaciales públicos no coordinados o que no están bien implementados.

13 Debilidad-3 (D3). No existe una continuidad de autoridades para la gestión del espacio público.
Mientras que los espacios públicos construidos hasta 1900 están protegidos por las autoridades mencionadas anteriormente, los espacios construidos después de 1900 carecen de una protección clara y falta de mantenimiento (bajo la administración del Instituto Nacional de Bellas Artes, encargada de la conservación y salvaguarda del patrimonio urbano arquitectónico a partir 1900, mientras que el Instituto Nacional de Antropología e Historia se encarga del patrimonio anterior a esta fecha). Estos espacios se encuentran en riesgo de ser modificados o incluso de ser privatizados parcial o completamente debido a la falta de instrumentación y recursos financieros. Un ejemplo es el Parque México, utilizado en 1985 y 2017 para el acopio de víveres y organización de actividades de rescate [Figura 4], donde algunos proyectos recientes de espacio público han estado ocupando espacio para estaciones de bicicletas con elementos fijos que limitan o evitan la movilidad emergente. ${ }^{10}$

A pesar de la existencia de diversos programas para mantener el espacio público en Ciudad de México, gran cantidad del territorio que se ha utilizado durante los desastres no se considera en dichos programas. Como consecuencia, dichos espacios quedan al margen de las mejoras u obras para adaptaciones. Así, se observa que no se realiza una superposición entre los instrumentos de planeación potencialmente útiles durante los desastres y las necesidades específicas que ofrecen otras medidas de mejoramiento. Por ejemplo, los programas de iluminación no están correlacionados con los espacios utilizados para refugios provisionales. Los programas de suministro de agua no están considerando la distribución de agua para casos de emergencia, etc. En el caso del programa de bajo-puentes, ${ }^{11}$ no se consideraron los usos y dichos espacios en gran medida se han privatizado. ${ }^{12}$

Ante esta situación, una gran debilidad es el hecho de que las autoridades cambian cada seis años y no hay continuidad para los proyectos propuestos o en progreso realizados por los anteriores departamentos. Un ejemplo es la Autoridad del Espacio Público (AEP), reemplazada por la Dirección de Patrimonio Cultural Urbano y de Espacio Público, que integró el espacio público y los asuntos patrimoniales. Esto se debe a las nuevas restricciones aplicadas al presupuesto oficial, pero esto también crea la necesidad de una reorganización interna de los departamentos, lo que hace que se dé una respuesta débil y deficiente a las necesidades emergentes, es decir, que tengan que comenzar de nuevo sus planes y la distribución de responsabilidades. Además, no existe un presupuesto suficiente para tales proyectos o bien, no existen directrices claras. Siendo ejemplo de esto la ahora extinta Agencia de Resiliencia de Ciudad de México, que propuso proyectos que vinculaban movilidad y desastres o la adaptación de espacios públicos para desastres pero que fue extinta. ${ }^{13}$ 


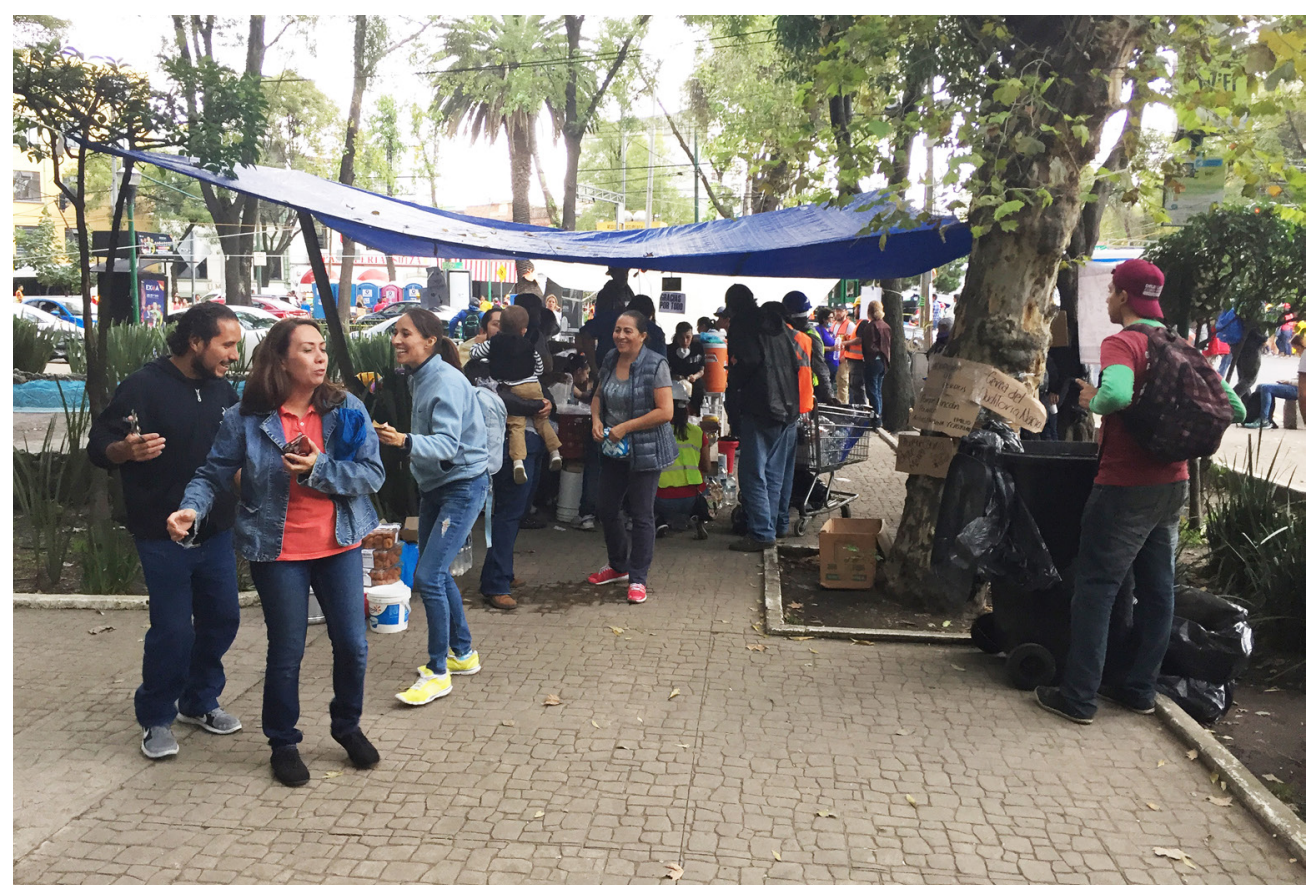

Figura 4

Parque México,

construido en 1927 sobre el trazo de una antigua pista de carreras de caballos y que es el elemento central de la colonia Hipódromo Condesa, Ciudad de México, ocupado para apoyar múltiples actividades en el sismo de septiembre 2017.

Fotografía: Autores.

\section{OPORTUNIDADES ${ }^{14}$}

14 Se entiende como oportunidad a las circunstancias externas y positivas que favorecen el uso y apropiación del espacio público en caso de emergencia.

15 Decreto emitido en el Diario Oficial de la Federación el 21 de octubre de 1985.

16 Oportunidad-1 (O1). Creación de nuevos espacios públicos después de desastres.
Como resultado del colapso de diversos edificios o su demolición necesaria, en 1985 se crearon nuevos espacios públicos, generalmente en el mismo lugar en el que habían estado los edificios. Esto siguió en parte al "Decreto de expropiación de propiedades urbanas en el Distrito Federal", 15 que fue creado con el propósito de expropiar propiedades dañadas para reconstruir la vivienda en el mismo lugar, además de la "regeneración urbana y la mejora de las propiedades expropiadas" (Magadán, 1987). Algunos de estos espacios adquirieron un significado de conmemoración y en ese lugar se colocaron esculturas o elementos relacionados con lo ocurrido. Otros espacios simplemente se dejaron como áreas verdes. Entre los espacios más significativos se encuentra la Plaza de la Solidaridad [Figura 5], un espacio en el que colapsaron el famoso Regis Hotel y el edificio de la Secretaría de Marina. ${ }^{16}$

Una pregunta importante es si actualmente aún existe oportunidad de crear nuevos espacios públicos vinculados a las nuevas condiciones comerciales, pues la apertura económica en el extranjero con el Tratado de Libre Comercio de 1988, promovió en México un cambio en las actividades de la industria manufacturera que provocó un proceso de desindustrialización. Las industrias se movieron hacia las periferias de la ciudad o incluso hacia otros territorios (Asuad, 2010), liberando grandes espacios. Al mismo tiempo se 

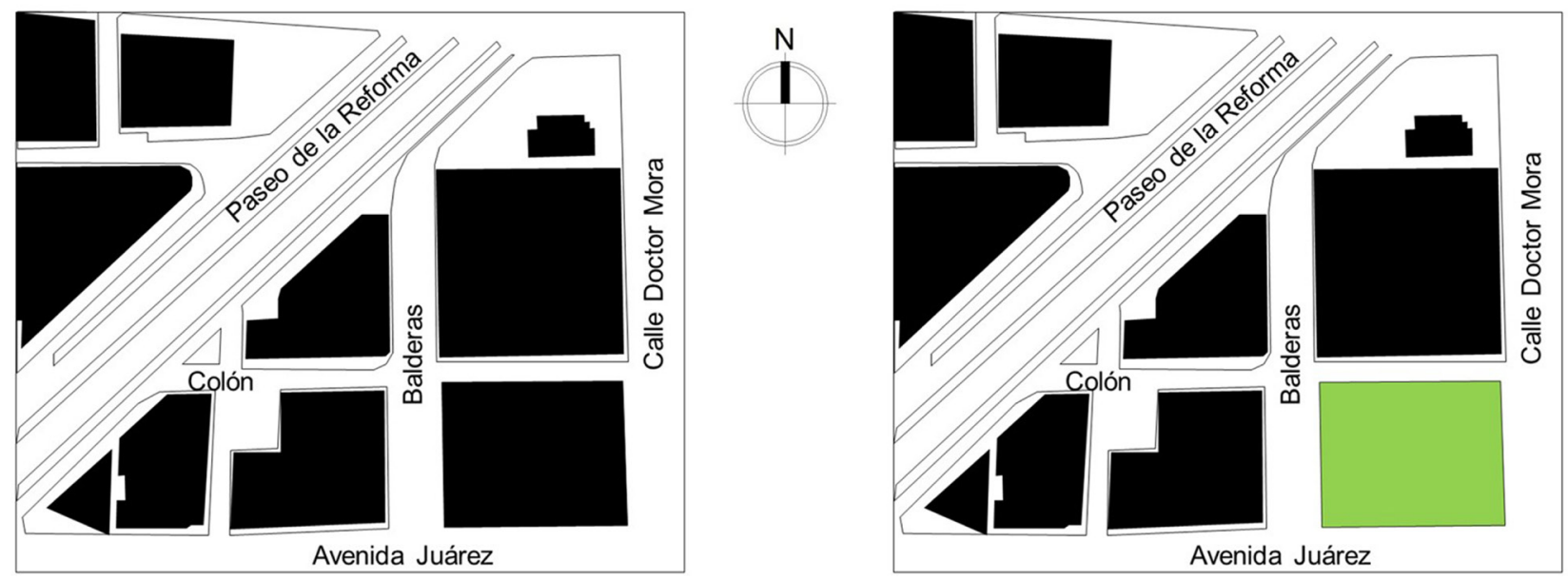

Figura 5

Creación de la Plaza de la Solidaridad por decreto de expropiación después del sismo de 1985 en Ciudad de México. Al centro de la plaza se ubica un monumento en memoria de las víctimas del desastre.

Fuente: Elaboración propia.

formó una importante centralidad despoblada, en parte debido al terremoto de 1985 y también al movimiento de la industria hacia la periferia, proceso que aún no concluye. Con este movimiento la reconfiguración de la ciudad se vio modificada, sobre todo debido a la oportunidad de suelo dentro de la ciudad. ${ }^{17}$

Para 2017, otro de los actores que desde el momento de la emergencia actuaron fueron las universidades. Después del terremoto de 2017 se han creado algunos comités dentro de las universidades ${ }^{18}$ para identificar y mitigar los riesgos dentro de cada institución. Como una oportunidad adicional, las autoridades están buscando una integración de dichos comités universitarios y muy importante es la inclusión que el Gobierno está haciendo de las universidades en los planes de reconstrucción y actualización de los códigos de construcción. Las universidades también han creado brigadas de emergencia para ofrecer ayuda humanitaria basada en las fortalezas de las diferentes disciplinas para promover la resiliencia, por ejemplo, grupos especializados para evaluar estructuras de edificios, analistas para reconstruir viviendas rurales o brigadas para ofrecer ayuda psicológica después de desastres. ${ }^{19}$

\footnotetext{
17 Oportunidad-2 (O2). La desindustrialización como una oportunidad para crear nuevos espacios públicos.

18 Por ejemplo el Comité Institucional de Seguridad y Resiliencia del Instituto Politécnico Nacional (IPN); el Comité Interno de Emergencias y Protección Civil de la Universidad Iberoamericana, o el Comité Interno de Protección Civil de la Universidad Nacional Autónoma de México (UNAM).

19 Oportunidad-3 (O3). Las universidades como recurso humano para la adaptación de espacios públicos ante desastres.
} 


\section{AMENAZAS 20}

20 Se entiende como amenaza a las situaciones adversas externas y negativas que tienen una probabilidad de ocurrencia, y que limitan o impiden el aprovechamiento, uso y apropiación del espacio público en caso de emergencia.

21 Amenaza-1 (A1). Incremento de la privatización del espacio público utilizado durante los desastres.

22 Amenaza-2 (A2). Nuevos conflictos sobre espacios públicos que dejó el desastre. 23 Amenaza-3 (A3). Verticalización indiscriminada.
Mientras que el terremoto de 1985 representó una oportunidad para crear nuevos espacios públicos, en el otro extremo estaban aquellos espacios públicos que desaparecieron o fueron privatizados. Uno de estos espacios fue el Estadio de Béisbol Delta, que pertenecía al Instituto Mexicano del Seguro Social (IMSS), y como espacio público institucional fue el lugar a donde los cuerpos de las víctimas fueron enviados durante el terremoto de 1985 , dado que los hospitales, cementerios e instalaciones funerarias, no eran suficientes ante la magnitud del terremoto (Monsiváis, 20I2, p. 72). A pesar de su valor histórico y social como equipamiento, la venta del estadio se anunció en 1999 y el último partido se jugó en ese estadio histórico en el año 2000. Posteriormente varias compañías lo adquirieron para construir allí un centro comercial (Excelsior, 20|4). ${ }^{21}$

En otros casos el destino de los espacios queda incierto, pues surgen nuevos conflictos por los nuevos espacios vacíos que deja el desastre. Este es el caso de Álvaro Obregón 286, un antiguo edificio de oficinas que se derrumbó en septiembre de 2017 con el lamentable fallecimiento de 49 personas. Después del terremoto el predio fue expropiado y el gobierno de Ciudad de México organizó un concurso de arquitectura para proponer un monumento que incluía un museo educativo y un memorial. Sin embargo, tal competencia encontró oposición del lado de las víctimas cuya vivienda fue destruida por el desastre, quienes expresaron que el presupuesto para tales proyectos debería utilizarse en la reconstrucción de viviendas y hasta ahora este conflicto no ha encontrado solución. ${ }^{22}$

La última amenaza la constituyen políticas urbanas que han inducido una verticalización indiscriminada, pues hacia principios del siglo XXI se contemplaron políticas públicas urbanas para redensificar la ciudad con viviendas sociales, como Bando 2 (norma suspendida en 2007), o Norma 26 (norma suspendida en 2013) para aprovechar la infraestructura y los servicios que actualmente están subutilizados y construir viviendas para las clases más vulnerables (Gobierno del Distrito Federal, 2000). Sin embargo, la especulación inmobiliaria estuvo presente en estos procesos creando nuevas áreas con densidades superiores a las normas, teniendo como consecuencia una transformación muy violenta del paisaje de la ciudad, pues donde había viviendas con 6 a 8 personas habitando, ahora aparecen edificios de 8 a 10 pisos para muchas familias con poco o nulo espacio público acorde a tales densidades, lo que hace a zla ciudad más vulnerable en caso de desastre, pues la relación habitantes/espacio público ha cambiado drásticamente considerando las necesidades en caso de evacuación. ${ }^{23}$ 


\section{ESTRATEGIAS BASADAS EN EL ANÁLISIS FODA}

24 Como el programa "Parques Públicos de Bolsillos", programa "Bajo puentes", programa "Lazos de amistad", programa "Ilumina tu ciudad", programa "Peatonalización de calles" y "Semipeatonalización de calles", programa de "Accesibilidad universal", entre otros. Con el objetivo común de rehabilitar el espacio público para el bien de la ciudadanía, además de las particularidades individuales de los programas.

25 (D2 / O3). Involucrar universidades para la producción de ideas de adaptación de espacios públicos ante desastres. 26 (D2 / O2-O3). Desarrollar programas mejor coordinados para la adaptación de los espacios públicos.

27 (F1-F3 / O3). Involucrar a las universidades, el Gobierno y la sociedad civil para la adaptación de los espacios públicos en relación con los monumentos históricos y modernos.

28 (F2-A3). Reforzamiento de directrices para la protección del patrimonio.

29 (D2 / A1). Fortalecer y coordinar mejor la protección del espacio público para que no se privaticen.
Hoy en Ciudad de México existen muchos programas ${ }^{24}$ para la recuperación, conservación y mejora de espacios públicos. Sin embargo, incluso en un territorio en riesgo, tales programas no están coordinados para ese objetivo. Esto abre posibilidades para una mejor coordinación y adaptación de dichos espacios. Esto podría ser posible y factible con una visión de integrar las necesidades necesarias, por ejemplo: seleccionar mejor el tipo de mobiliario urbano (pensando también en usos emergentes), mejorar la accesibilidad (también para emergencias y no solo para las necesidades cotidianas), mejorar las comunicaciones, una flora adecuada, árboles para crear sombras, instalaciones de agua diseñadas estratégicamente, iluminación de emergencia, etc. En la producción de ideas para esta adaptación, la participación de escuelas de arquitectura sería muy útil [Figura 6]. ${ }^{25}$ En otras palabras, una integración de los ejes de infraestructura a partir de nuevas ideas sigue siendo muy importante en el camino hacia la adaptación. ${ }^{26}$

Dentro de las acciones propuestas, las autoridades a cargo de la protección del patrimonio podrían fortalecer las pautas para evaluar los proyectos de densificación contra la capacidad existente de los espacios públicos del patrimonio. ${ }^{27}$ Es decir, ser más conscientes de la relación densidad / área. Un ejemplo en Ciudad de México es el Parque México, antes mencionado, que obviamente no aumenta su superficie, pero el entorno se ha densificado en las últimas décadas como consecuencia del crecimiento urbano vertical. ${ }^{28}$ En este sentido, será fundamental respetar y adoptar los indicadores internacionales como los de la Organización Mundial de la Salud con respecto a la cantidad de metros cuadrados que un ser humano necesita para vivir en una ciudad (la OMS indica entre II y 13 metros cuadrados por habitante, y en Ciudad de México son menos de cinco). ${ }^{29}$
Figura 6

Detalle de una propuesta de adaptación de espacios públicos institucionales para ser ocupados como refugio después de un desastre. La propuesta emana de un concurso que los autores co-organizaron con estudiantes de arquitectura del Instituto Politécnico Nacional en junio de 2019 .

Fuente: Cortesía de

la autora Eloísa García Martínez. 

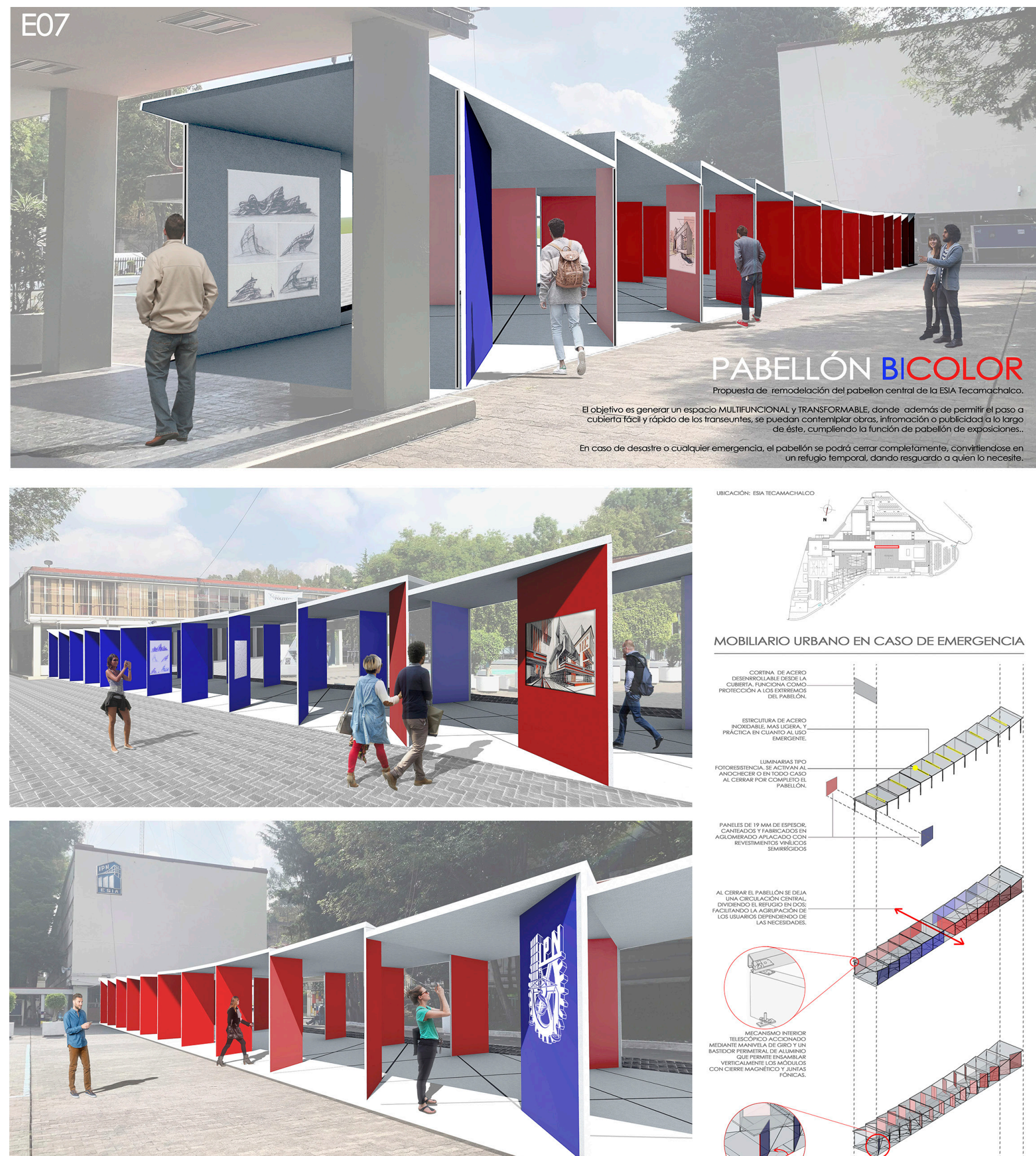

MOBILIARIO URBANO EN CASO DE EMERGENCIA
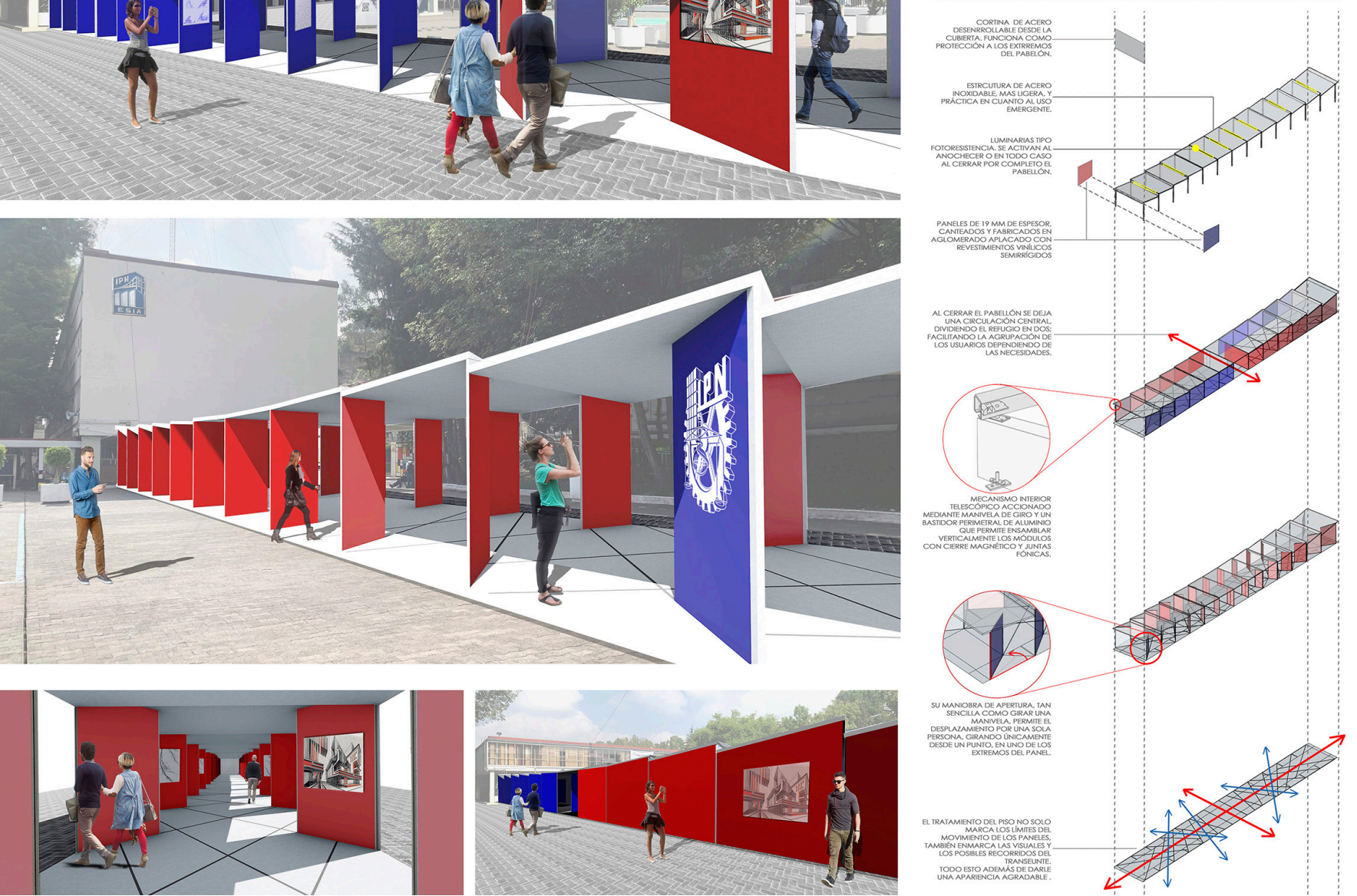
30 (F3-A1) Regulación del uso de espacios públicos ya privatizados como parte de los programas de recuperación.

31 (F3 / O3). Preparar espacios públicos para desastres en áreas más específicas.

32 (F3 / O2). Definir proyectos en zonas desindustrializadas de acuerdo con la naturaleza sísmica del área.

33 (D3-A3). Un organismo independiente de los períodos políticos.

34 (D2 / O2). Coordinar acciones entre municipios.

35 (D3 / A1-A3). Fortalecer la opinión pública para defender el espacio público y evitar la verticalización indiscriminada.

36 (F3-A2). Establecimiento de un diálogo más organizado entre la sociedad civil y las autoridades.
Para los espacios públicos ya privatizados, el uso del suelo podría regularse, pero con el objetivo de tener un efecto sobre el tipo de negocio que se aprovechará en caso de desastre, para que el negocio pueda apoyar planes de emergencia. Por ejemplo, en bajo-puentes ubicados a lo largo del anillo interior de la ciudad se han estado alquilando espacios para negocios de comida, tiendas de diseño y distribuidores de automóviles; sin embargo, se podría llegar a un acuerdo para que estas empresas en caso de desastre, puedan ofrecer a la población sus servicios o productos: alimentos gratuitos en caso de emergencia, herramientas de construcción para facilitar el rescate de las víctimas (cascos, guantes industriales, picos y palas), o los negocios de grandes superficies como bares podrían convertirse en refugios provisionales. En 2017, varias empresas ya asumieron esta responsabilidad, pero podría ser posible una estrategia más organizada. ${ }^{30}$

En cuanto a la desindustrialización, se pueden proponer nuevas normas para indicar una cantidad mínima de espacio público para proyectos urbanos a gran escala, particularmente en áreas donde este proceso aún está en progreso y donde todavía existe la oportunidad. Además, un grupo asesor, compuesto por expertos en planificación, geología, geografía, políticas públicas y economía, podría encontrar con los desarrolladores inmobiliarios un equilibrio entre el desarrollo urbano y la seguridad..$^{31}$ En las áreas ya desindustrializadas, los proyectos de acciones emergentes pueden implementarse con el objetivo de adaptar espacios para desastres o expropiar predios en zonas desindustrializadas para la creación de espacios públicos. ${ }^{32}$

Finalmente, para evitar la falta de continuidad en estos proyectos, sería fundamental promover la creación de un órgano de gobierno que tenga voz y voto en proyectos urbanos y transformaciones que afecten la verticalización y desaparición del espacio público y el espacio potencialmente público, que son los espacios que deja la desindustrialización, así como el uso de espacios públicos institucionales, ya sean centros deportivos o plazas de las alcaldías. Este organismo debería existir de forma paralela e independiente de los cambios políticos o períodos de las administraciones públicas ${ }^{33}$ y podría abarcar diferentes jurisdicciones. ${ }^{34}$ Estas decisiones deberán tomarse junto con la sociedad civil, ${ }^{35}$ sobre todo en lo que concierne a los nuevos usos de los predios vacíos ocasionados por desastres. ${ }^{36}$

Se identificaron amenazas y debilidades, pero al mismo tiempo fortalezas y oportunidades en todos los niveles y circunstancias. Se requiere más inversión para el desarrollo de tales estrategias y la realización de estudios prospectivos que apoyen la creación de una conciencia referida anteriormente para llevar a cabo las estrategias identificadas, esto requeriría una conciencia general de los riesgos involucrados al vivir en un territorio vulnerable principalmente a amenazas de tipo sísmico. Nos referimos tanto a las autoridades como a la población en general, así 


\section{CONCLUSIONES}

AS / Vol. 38. N57 / ENERO 2020 // ISSN impresa 07 | 6-2677 / ISSN digital 0719-6466

LA HABITABILIDAD EFÍMERA:

EL ESPACIO PÚBLICO COMO REFUGIO DE DESASTRES EN CIUDAD DE MÉXICO

Milton Montejano Castillo / Mildred Moreno Villanueva como a las empresas y otros actores involucrados en la construcción y transformación de la ciudad, pues a pesar de los efectos que puede traer un desastre, estos pueden brindar oportunidades para hacer que una ciudad sea menos vulnerable, ya que se sabe que estos desastres continuarán. En estos procesos es importante identificar los grupos ya organizados a escala del vecindario, para ofrecerles más herramientas y conocimientos para que puedan organizarse mejor a su favor para la defensa y voz de sus derechos en las instituciones públicas responsables de la autorización de proyectos urbanos, y proyectos para la reducción del riesgo de desastres.

Se identificaron muchas amenazas y debilidades, pero al mismo tiempo hay muchas fortalezas y oportunidades en todos los niveles y circunstancias, como el desarrollo tecnológico actual, que deberían usarse de manera más efectiva. Se requiere más inversión para el desarrollo de tales estrategias y la realización de estudios prospectivos que apoyarían la creación de conciencia referida anteriormente para llevar a cabo las estrategias identificadas, esto requeriría una conciencia general de los riesgos involucrados en vivir en un territorio vulnerable principalmente a la amenaza sísmica e hidrometeorológica (no tratadas en este artículo). Nos referimos tanto a las autoridades como a la población en general, así como a las empresas y otros actores involucrados en la construcción y transformación de la ciudad.

A pesar de los efectos que puede traer un desastre, puede brindar oportunidades para hacer que una ciudad sea menos vulnerable a futuros desastres, ya que se sabe que estos continuarán ocurriendo. Asimismo, hay ejemplos en algunas partes del mundo que se pueden aprender, pero hay otros tipos de amenazas y limitaciones que no son necesariamente de origen natural, nos referimos a aquellos que podrían estar relacionados con aspectos culturales y socioeconómicos, aspectos que limitan la identificación de oportunidades y, por lo tanto, dificultan pensar y visualizar oportunidades y acciones de mitigación. Finalmente, con base en las tendencias y estrategias identificadas, estos resultados pueden usarse para identificar escenarios probables si no actuamos a tiempo, o los deseables si actuamos a tiempo.

Existen ejemplos en algunas partes del mundo de los que se puede aprender en cuanto a una mejor gestión del riesgo de desastres. Países como Japón han estado adaptando ciudades a los desastres como parte del proceso de reconstrucción. Por ejemplo, la recuperación y adaptación de grandes parques urbanos preparados para funcionar como refugios de emergencia durante un mínimo de 72 horas. Ejemplo de esto es el Parque Minato No Mori, un proyecto a gran escala ubicado en Kobe, Japón, preparado para recibir víctimas en caso de desastre y con sistemas de alcantarillado 
pre-instaladas, iluminación, celdas solares, agua potable y zonas verdes con frutas y verduras comestibles para apoyar la supervivencia de los habitantes. Ese parque fue rehabilitado después del Gran Terremoto de Hanshin en 1995 en Kobe sobre una antigua zona industrial. Existen otros ejemplos a escala del barrio como el caso del barrio Matsumoto, en Kobe, Japón, que contiene una serie de parques de bolsillo en los que los residentes locales, con el apoyo del gobierno, se han estado adaptando para ser utilizados como un recurso en la secuela del desastre. Dichos parques de bolsillo tienen pizarras para información, rutas de evacuación, un reloj, un almacén con algunas herramientas como picos y palas, equipos, toldos que se desenrollan y que sirven como paredes de refugios provisionales, bombas de agua, e iluminación para sobrevivir los primeros tres días antes de que la ayuda externa llegue al lugar.

Pero también hay otros tipos de amenazas y limitaciones que no son necesariamente de origen natural, nos referimos a aquellos que podrían estar relacionados con aspectos culturales y socioeconómicos, aspectos que limitan la identificación de oportunidades $y$, por lo tanto, dificultan pensar y visualizar oportunidades y acciones de mitigación. Con base en las tendencias y estrategias identificadas, estos resultados pueden usarse para definir escenarios probables -si no actuamos a tiempo-, o escenarios deseables y posibles, si se crean las acciones correspondientes, solo así se construirán espacios públicos más habitables y seguros para el futuro. 


\section{BIBLIOGRAFÍA}

